\title{
Complexity of Some Geometric and Topological Problems
}

\author{
Marcus Schaefer ${ }^{\star}$ \\ DePaul University, Chicago, IL 60604, USA \\ mschaefer@cs. depaul.edu
}

\begin{abstract}
We show that recognizing intersection graphs of convex sets has the same complexity as deciding truth in the existential theory of the reals. Comparing this to similar results on the rectilinear crossing number and intersection graphs of line segments, we argue that there is a need to recognize this level of complexity as its own class.
\end{abstract}

\section{Introduction}

We show that determining whether a graph can be realized as an intersection graph of convex sets in the plane has the same complexity as deciding the truth of existential first-order sentences over the real numbers. This connection between geometry and logic is not uncommon: Kratochvíl and Matoušek [11, for example, showed that recognizing intersection graphs of line segments also has the same complexity as the existential theory of the reals (we include a slightly simplified proof of that result), and there are several other geometric problems that share the same complexity. We therefore suggest the introduction of a new complexity class $\exists \mathbb{R}$, which captures the complexity of deciding the truth of the existential theory of the reals.

Remark 1. In the formal definition of $\exists \mathbb{R}$ we will not allow equality. If we define $\exists=\mathbb{R}$ like $\exists \mathbb{R}$, but with equality allowed, we obtain a complexity class for which there is a name in the Blum-Shub-Smale model of computing over the reals: $\operatorname{BP}\left(\mathbf{N P}_{\mathbb{R}}^{0}\right)$ 3]; this class has not played a major role in that model so far (as reflected by the complexity of the notation). Somewhat surprisingly, $\exists \mathbb{R}=\exists=\mathbb{R}\left[21\right.$, even though algebraically the two classes differ, e.g. $x^{2}=2$ defines an irrational point, which is not possible without equality.

The first combinatorial problem shown complete for $\exists \mathbb{R}$ was stretchability of simple pseudoline arrangements, a result due to Mnëv as a byproduct of his universality theorem [14]18/23. There have been several other problems classified as $\exists \mathbb{R}$-complete since, including the algorithmic Steinitz problem 2, intersection graphs of line segments [11, and straight-line realizability of abstract topological graphs [13]. Very often, however, $\exists \mathbb{R}$-completeness is not claimed explicitly;

\footnotetext{
* Some of this work was done in the beautiful library at Oberwolfach during the seminar on Discrete Geometry in September 2008.
} 
for example, in the case of the rectilinear crossing number, Bienstock gave a reduction from stretchability to the rectilinear crossing number problem. Since the problem can easily be shown to lie in $\exists \mathbb{R}$ (see Section 3) computing the rectilinear crossing number is $\exists \mathbb{R}$-complete. So - in a sense - the complexity of the problem is known precisely, but it is not unusual to see the complexity question for the rectilinear crossing number listed as an open problem [15]. There is some good reason for that: we do not know how to capture $\exists \mathbb{R}$ well with respect to classical complexity classes: we know that it contains NP (this follows easily from the definition of $\exists \mathbb{R}$; also, Shor gave a direct proof that stretchability is NPhard 23] ) and is itself contained in PSPACE, a remarkable improvement on Tarski's original decision procedure for the theory of reals by Canny 4 . So, in a sense, we do not know the complexity of the rectilinear crossing number problem, since we can only position it between NP and PSPACE. We should approach this situation in the same spirit as we do NP-completeness: NP-completeness of a problem does not exclude the possibility that the problems is in $\mathbf{P}$ or $\mathbf{E X P}$ complete, but proving it NP-complete focuses that question on the real issue, away from the particular problem, and towards the study of the structural aspects of NP-completeness as a whole. Something similar should be possible for $\exists \mathbb{R}$-completeness. Knowing that a problem is $\exists \mathbb{R}$-complete does not tell us more than that it is NP-hard and in PSPACE in terms of classical complexity, but it does tell us where to start the attack: by studying the structure of $\exists \mathbb{R}$-complete problems; so asking, like 15, whether the rectilinear crossing number can be decided in NP is really asking whether $\exists \mathbb{R}$ lies in NP. And that puts a different perspective on the problem. A solution will likely not come out of graph drawing or graph theory, but out of a better understanding of real algebraic geometry and logic; what satisfiability is for NP, the existential theory of the reals is for $\exists \mathbb{R}$.

To justify our claim of the importance of $\exists \mathbb{R}$ and the necessity of a new complexity class, we need to find natural $\exists \mathbb{R}$-complete problems. In this note we give three examples: two known (one implicitly), one new. Plus one bonus problem in topological inference. This work is part of a more comprehensive project in which we survey many other problems as candidates for $\exists \mathbb{R}$-completeness including several other new results, including graph and linkage realizability and the complexity of finding Brouwer fixed points and Nash equilibria [19].

\section{Background}

The existential theory of the reals is the set of true sentences of the form

$$
\left(\exists x_{1}, \ldots, x_{n}\right) \varphi\left(x_{1}, \ldots, x_{n}\right),
$$

where $\varphi$ is a quantifier-free Boolean formula (without negation) over the signature $(0,1,+, *,<)$ interpreted over the universe of real numbers. It was first shown by Tarski that this theory is decidable; it is now known to be decidable in PSPACE by a result of Canny [4]. 
By disallowing negation, we restrict ourselves to strict inequalities, which is the version of the problem relevant to the examples presented in the current note; let us call the set of true sentences of this theory STRICT INEQ. With this we define the complexity class $\exists \mathbb{R}$ as the closure of STRICT INEQ under polynomial-time reductions. A problem is $\exists \mathbb{R}$-complete if it belongs to $\exists \mathbb{R}$ and every problem in $\exists \mathbb{R}$ can be reduced to it by a polynomial-time reduction. Note that $\mathbf{N P} \subseteq \exists \mathbb{R}$, since we can express satisfiability of a Boolean formula in $\exists \mathbb{R}$. For example, $(x \vee \neg y \vee z) \wedge(\neg x \vee y \vee z) \wedge(\neg x \vee \neg y \vee \neg z)$ is equivalent to

$$
\begin{aligned}
& (\exists x, y, z)[(-\varepsilon<x<2) \wedge(-\varepsilon<y<2) \wedge(-\varepsilon<z<2) \\
& \wedge(x(1-y) z)+((1-x) y z)+((1-x)(1-y)(1-z))<\varepsilon],
\end{aligned}
$$

if we choose $\varepsilon=1 / 8(1+4 m)=1 / 104$ where $m$ is the number of clauses, so $m=3$ in the example.

A pseudoline is a simple closed curve in the projective plane that is homeomorphic to a straight line. An arrangement of pseudolines is a collection of pseudolines so that each pair of pseudolines cross at most once (and do not touch). An arrangement is simple if no more than two pseudolines pass through a point. Two arrangements are equivalent if there is a homeomorphism of the projective plane turning one into the other. An arrangement of pseudolines is simply stretchable if it is equivalent to a simple arrangement of straight lines. (So being simply stretchable means the original arrangement is simple and stretchable.)

Remark 2. If one wants to avoid the reference to the projective plane, one can define pseudolines in the plane as simple $x$-monotone curves, that is curves that cross every vertical line exactly once. If one takes this route, one needs to require that in an arrangement of pseudolines every pair of pseudolines crosses exactly once (as opposed to at most once).

Mnëv showed that STRICT INEQ reduces to SIMPLE STRETCHABILITY; since the reverse is also true, SIMPLE STRETCHABILITY is $\exists \mathbb{R}$-complete. Shor later simplified the reduction [23. From this it immediately follows that SIMPLE STRETCHABILITY is NP-hard, since $\exists \mathbb{R}$-hardness implies $\mathbf{N P}$-hardness as we saw above. (Shor 23] also gave a direct proof.)

$\exists \mathbb{R}$-hard problems typically require large representations; Goodman, Pollack and Sturmfels [8] showed that there are stretchable arrangements of $n$ pseudolines whose coordinate representation requires $2^{c n}$ bits for some constant $c>0$. (Equivalently, if we want to draw the endpoints on a grid, it must have size at least $2^{2^{c^{\prime} n}}$ for some $c^{\prime}>0$.) Typically, reductions from an $\exists \mathbb{R}$-hard problem $A$ to another problem $B$ are geometric in the sense that if we are given a geometric representation of $B$, we can derive a geometric representation of $A$ which is of at most polynomial size in the bit-size of the original representation. For example, this is the case for Bienstock's reduction from simple stretchability to rectilinear crossing number. We can then conclude (as Bienstock did) that there are graphs for which any straight-line drawing with optimal rectilinear crossing number requires $2^{c n}$ bits of storage. All other reductions in this note are also geometric, so geometric representations of these problem will require exponential precision. 


\section{Rectilinear Crossing Number}

The rectilinear crossing number of $G$, lin-cr $(G)$, is the smallest number of crossings in a straight-line drawing of $G$, that is, a drawing in which every edge is represented by a straight-line segment and at most two edges intersect in a point. The problem is NP-hard by Garey and Johnson's original proof that computing the crossing number is NP-hard [7] and it remains $\mathbf{N P}$-hard even if the graph is cubic and 3-connected 916. Bienstock gave an easy and elegant reduction that shows that SIMPLE STRETCHABILITY reduces to deciding whether lin-cr $(G) \leq k$, even if $G$ is restricted to be cubic [1].

Theorem 1 (Bienstock [1]). Computing the rectilinear crossing number of a (cubic) graph is $\exists \mathbb{R}$-complete. There are graphs for which the coordinates of the vertices in an lin-cr-optimal drawing of the graph require exponential precision (in the size of the graph).

Proof. $\exists \mathbb{R}$-hardness follows from Bienstock's reduction as does the claim about exponential precision, so we only have to show that determining whether lin-cr $(G) \leq k$ lies in $\exists \mathbb{R}$; the only, small, difficulty is that we do not know which edges of the graph cross, so we need to guess a subset of pairs of edges of size at most $k$ using real numbers.

Using quantifier-free formulas, we can define $\overline{\text { colinear }}\left(x_{1}, y_{1}, x_{2}, y_{2}, x_{3}, y_{3}\right)$ to express that the three points $\left(x_{i}, y_{i}\right)_{i \in[3]}$ are not colinear and a predicate $\overline{\operatorname{cross}}\left(x_{1}, y_{1}, x_{2}, y_{2}, x_{1}^{\prime}, y_{1}^{\prime}, x_{2}^{\prime}, y_{2}^{\prime}\right)$ expressing that the two line segments determined by $\left(x_{1}, y_{1}\right),\left(x_{2}, y_{2}\right)$ and $\left(x_{1}^{\prime}, y_{1}^{\prime}\right),\left(x_{2}^{\prime}, y_{2}^{\prime}\right)$ do not have a point in common (details in the full paper).

For a fixed $k$ and $m=|E(G)|$, we can write a predicate $\operatorname{atmost}_{k}\left(z_{1}, \ldots, z_{m^{2}}\right)$ which guarantees that at most $k$ of the $z_{i}$ are greater than zero:

$$
\bigwedge_{i \in\left[m^{2}\right]}\left(\left(-1 / 2 m^{4}<z_{i}<0\right) \vee\left(1+1 / 2 m^{2}<z_{i}\right)\right) \wedge \sum_{i \in\left[m^{2}\right]} z_{i}<k+1 .
$$

Since lin-cr $(G) \leq\left(\begin{array}{c}m \\ 2\end{array}\right)$, we can assume that $k \leq\left(\begin{array}{c}m \\ 2\end{array}\right)$; so the sum of the negative $z_{i}$ is at least $-1 / 2 m^{2}$. If more than $k$ of the $z_{i}$ are positive, their sum is at least $k+1+(k+1) / 2 m^{2}$, but then the total sum is at least $k+1$. On the other hand, given any subset of the $z_{i}$ of size at most $k$, we can assign each $z_{i}$ in the set the value $1+2 / 3 m^{2}$ and every other $z_{i}$ gets the value $-2 / 3 m^{4}$, so that $\sum_{i \in\left[m^{2}\right]} z_{i} \leq k+2 / 3<k+1$, showing that any subset of the $z_{i}$ can be realized by atmost $k$.

With these predicates, we can express $\operatorname{lin}-\operatorname{cr}(G) \leq k$; to simplify the formula, suppose that $V(G)=[n], E(G)=[m]$, and we have two functions $h, t: E \rightarrow V$ so that $h(e)=x$ and $t(e)=y$ if $e \in E$ is an edge between $x, y \in V$. We use $z_{(i-1) m+j}>0$ to indicate that edges $i$ and $j$ are allowed to cross. Now $\operatorname{lin}-\operatorname{cr}(G) \leq k$ if and only if 


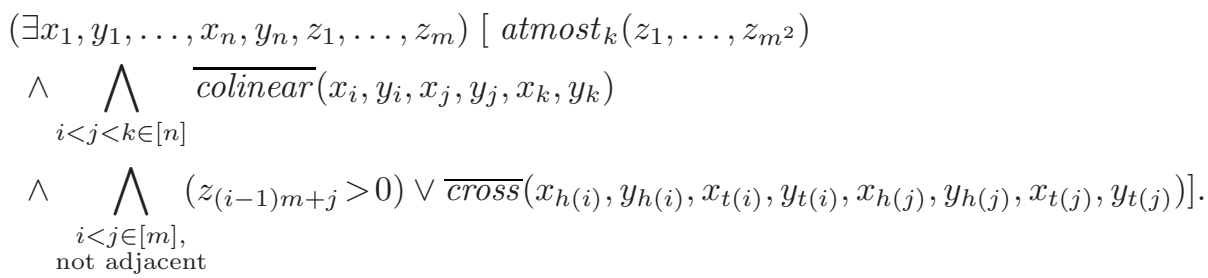

\section{Intersection Graphs of Segments}

$G=(V, E)$ is an intersection graph of line segments if for each $v \in V$ there is a line segment $\ell_{v}$ in the plane so that $u v \in E$ if and only if $\ell_{u}$ and $\ell_{v}$ intersect.

Theorem 2 (Kratochvíl, Matoušek [11]). Recognizing intersection graphs of line segments is $\exists \mathbb{R}$-complete. There are graphs for which the coordinates of the endpoints of the line segments in any intersection representation of the graph require exponential precision (in the size of the graph).

Remark 3. Kratochvíl and Pergel showed that the recognition of intersection graphs of line segments remains NP-hard if the graphs have girth at least $k$ for any fixed $k[12$. Can this be extended to $\exists \mathbb{R}$-completeness?

We give a slightly simplified proof of Theorem 2 the argument will also be used in Theorem 3. We write $[n]$ for $\{1, \ldots, n\}$.

Lemma 1. Suppose we have Jordan curves $\ell,\left(\ell_{i}\right)_{i \in[n]},\left(s_{i}^{j}\right)_{i \in[n-1], j \in[3]}$, and $\left(c_{i}\right)_{i \in[4 n]}$ in the plane so that

(i) $\ell$ crosses $\ell_{i}, i \in[n]$, and $s_{i}^{2}, i \in[n-1]$,

(ii) $c_{i}$ crosses $c_{i+1}\left(c_{1}\right.$ for $\left.i=4 n\right)$ exactly once, $i \in[4 n]$,

(iii) $\ell_{i}$ crosses $c_{2 i}$ and $c_{4 n-2 i+2}, i \in[n]$,

(iv) both $s_{i}^{1}$ and $s_{i}^{3}$ cross $s_{i}^{2}, i \in[n-1]$,

(v) $s_{i}^{1}$ crosses $c_{2 i+1}$ and $s_{i}^{3}$ crosses $c_{4 n-2 i+1}, i \in[n-1]$,

(vi) the only other crossings among these curves are between pairs of $\ell_{i}$.

Then the curves $\ell_{i}$ cross $\ell$ either in order $\ell_{1}, \ldots, \ell_{n}$ or in the reverse of that order. The conclusion remains true if instead of $(i)$ we only require that $\left(i^{\prime}\right) \ell$ crosses $\ell_{i}, i \in[n]$, and $\left(i^{\prime \prime}\right) s_{i}^{2}, i \in[n-1]$, may cross $\ell$, but it does lie in the same connected component of $\mathbb{R}^{2}-\cup_{i \in[4 n]} c_{i}$ as $\ell$.

We call the collection of curves $\left(s_{i}^{j}\right)_{i \in[n-1], j \in[3]}$, and $\left(c_{i}\right)_{i \in[4 n]}$ and the way they cross each other and the curves $\ell$ and $\left(\ell_{i}\right)_{i \in[n]}$ the ordering gadget for $\ell$ with respect to $\left(\ell_{i}\right)_{i \in[n]}$. The intended drawing of the curves of the lemma is shown in Figure 1, but there are other drawings.

Proof. The set $\bigcup_{i \in[4 n]} c_{i}$ contains a (unique) closed Jordan curve $C$. $C$ separates the plane into two faces; without loss of generality (since we are dealing with 


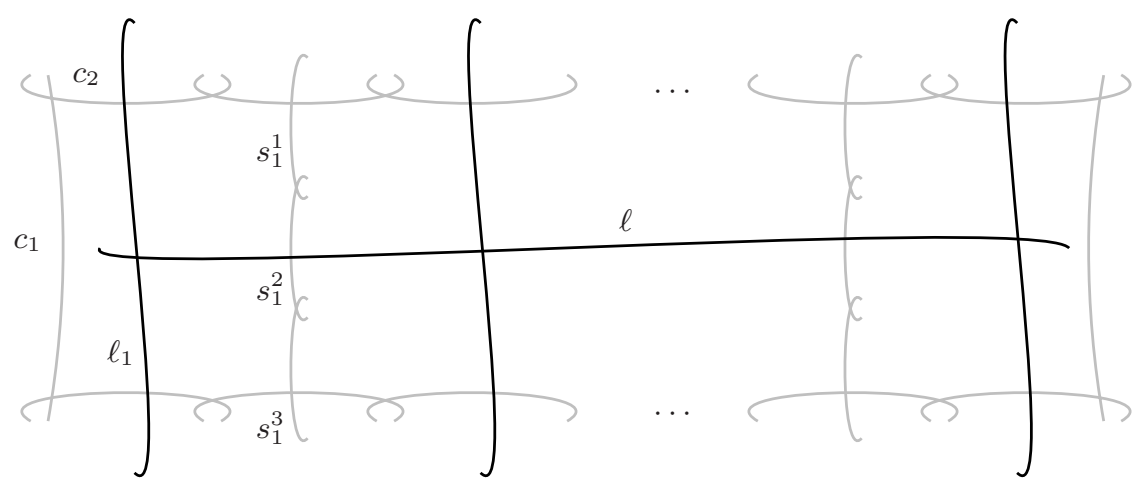

Fig. 1. An ordering gadget

curves), we may assume that $\ell$ lies in the inner face. Since $\ell$ crosses every $s_{i}^{2}$, and these curves do not cross any $c_{j}$, all the $s_{i}^{2}$ also lie in the inner face of $C$ (indeed, $\left(i i^{\prime}\right)$ is sufficient to draw this conclusion). Within each $S_{i}:=c_{2 i+1} \cup s_{i}^{1} \cup s_{i}^{2} \cup$ $s_{i}^{3} \cup c_{4 n-2 i+1}, i \in[n-1]$ choose a Jordan arc $s_{i}$ with endpoints on $C$. The $s_{i}$ are chords of $C$ that lie in the inner face of $C$ (since $s_{i}^{2}$ does); moreover, the $s_{i}$ do not intersect each other (since any two $S_{i}$ are disjoint) or any of the $\ell_{i}$ (since $S_{i}$ and $\ell_{j}$ are disjoint for all $\left.i, j \in[n]\right)$. Now the ends of the $s_{i}$ and $\ell_{i}$ along $C$ are in order $\ell_{1}, s_{1}, \ell_{2}, \ldots, s_{n-1}, \ell_{n}, \ell_{n}, s_{n-1}, \ldots, s_{1}, \ell_{1}$ (up to cyclic shifts). Since every $\ell_{i}$ has to cross $\ell$ and has to do so within $C$, it must do so in order $\ell_{1}, \ldots, \ell_{n}$ or its reverse.

Proof (Theorem Q . It is easy to see that the problem lies in $\exists \mathbb{R}$. To show $\exists \mathbb{R}$ hardness, we reduce from SIMPLE STRETCHABILITY. Suppose we are given a simple arrangement $\mathcal{A}$ of pseudolines. Remark 2 allows us to think of the arrangement as a set of simple, $x$-monotone curves.

Add a triangle $T$ formed by three pairwise crossing curves so that all crossings of curves in $\mathcal{A}$ lie within the region enclosed by $T$ and one edge of $T$ crosses all curves in $\mathcal{A}$ (for example, choose a vertical line segment to the left of all crossings in $\mathcal{A}$ that is long enough to cross every curve in $\mathcal{A}$ ). We can choose $T$ so that we know the order of crossings of curves belonging to $\mathcal{A}$ with the curves of $T$.

Cut off the pseudolines just beyond the boundary of $T$ and let $\mathcal{C}$ contain all the resulting curves together with the three curves from $T$. For each curve $\ell \in \mathcal{C}$ add the ordering gadget - as constructed in Lemma 1 - with respect to all remaining curves in $\mathcal{C}$. Also, require that curves $c_{2 i}$ and $c_{4 n-2 i+2}$ for $\ell$ cross the corresponding two $c$-curves of $\ell_{i}$ (see Figure 2). Let $G_{\mathcal{A}}$ be the resulting intersection graph of all curves.

In any (curvilinear) drawing of $G_{\mathcal{A}}$, the order of crossings along each curve from $\mathcal{A}$ and $T$ with curves from that set is as in the original arrangement or reversed by Lemma 1, since we added ordering gadgets for each of those curves. In particular, the crossings with $T$ are first and last along each curve from $\mathcal{A}$, and therefore all crossings between curves of $\mathcal{A}$ occur within the region enclosed by 
$T$. Now the crossings of $\mathcal{A}$ with the edge of $T$ that crosses all curves in $\mathcal{A}$ occur either in the original order or in the reversed order. However, this means that the order of crossings along edges realizing $\mathcal{A}$ must either all be in the original order, or all of them are reversed. Hence, if $G_{\mathcal{A}}$ can be realized as an intersection graph of straight-line segments, then $\mathcal{A}$ is stretchable.

It is easy to see that if the original arrangement $\mathcal{A}$ is stretchable, then so is the extended arrangement (the intended drawing of the ordering gadget is shown in Figure 10.

Finally, the reduction is geometric, so the claim about exponential precision follows.

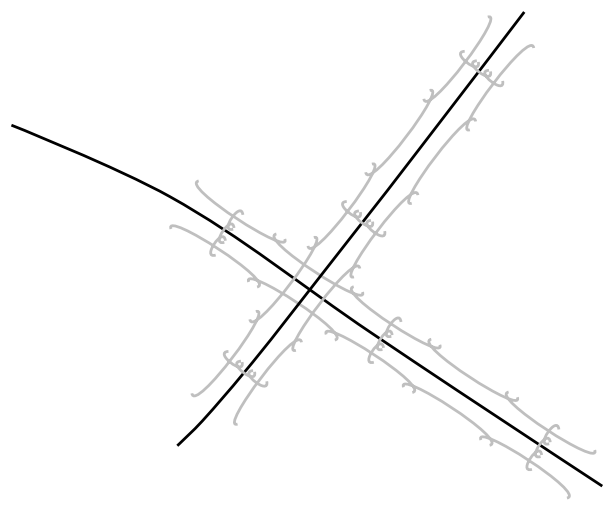

Fig. 2. Two arrangement lines crossing, with gadgets

\section{Intersection Graphs of Convex Sets}

$G=(V, E)$ is an intersection graph of convex sets if for every $v \in V$ there is a convex set $C_{v}$ in the plane so that $u v \in E$ if and only if $C_{u}$ and $C_{v}$ intersect. We say two regions in the plane intersect if they share a common point. The problem is known to be in PSPACE and NP-hard [1].

Theorem 3. Recognizing intersection graphs of convex sets is $\exists \mathbb{R}$-complete. There are graphs for which any realization as intersection graphs of convex polygons requires exponential precision in writing down the coordinates of the vertices of the polygon 1

For the $\exists \mathbb{R}$-hardness proof we carefully adapt the reduction from SIMPLE STRETCHABILITY to SEG we saw in Theorem 2, and we begin by restating Lemma 1 for convex sets.

${ }^{1}$ The result on exponential precision has been independently obtained by Martin Pergel[10. 
Lemma 2. Suppose we have convex sets $L,\left(L_{i}\right)_{i \in[n]},\left(S_{i}^{j}\right)_{i \in[n-1], j \in[3]}$, and $\left(C_{i}\right)_{i \in[4 n]}$ in the plane so that

(i) $L$ intersects $L_{i}, i \in[n]$, and $S_{i}^{2}, i \in[n-1]$,

(ii) $C_{i}$ intersects $C_{i+1}\left(C_{1}\right.$ for $\left.i=4 n\right), i \in[4 n]$,

(iii) $L_{i}$ intersects $C_{2 i}$ and $C_{4 n-2 i+2}, i \in[n]$,

(iv) both $S_{i}^{1}$ and $S_{i}^{3}$ intersect $S_{i}^{2}, i \in[n-1]$,

(v) $S_{i}^{1}$ intersects $C_{2 i+1}$ and $S_{i}^{3}$ intersects $C_{4 n-2 i+1}, i \in[n-1]$,

(vi) the only other intersections among these regions are between pairs of $L_{i}$.

Moreover, suppose we have Jordan curves $\ell$ in $L$ and $\ell_{i}$ in $L_{i}, i \in[n]$ so that every $\ell_{i}$ crosses $\ell$. Then the order of the intersections along $\ell$ is either $\ell_{1}, \ldots, \ell_{n}$ or the reverse of that order.

We call the collection of convex sets $\left(S_{i}^{j}\right)_{i \in[n], j \in[3]}$, and $\left(C_{i}\right)_{i \in[8 n-4]}$ and the way they intersect each other and the sets $L$ and $\left(L_{i}\right)_{i \in[n]}$ the ordering gadget for $L$ with respect to $\left(\ell_{i}\right)_{i \in[n]}$. The intended drawing of the convex sets is similar to the one shown in Figure 1 with line segments replaced by convex sets.

Proof. Pick vertices $v_{i} \in C_{i} \cap C_{i+1}, i \in[4 n-1]$, and $v_{4 n} \in C_{4 n} \cap C_{1}$, and let $c_{i}$ be a straight-line segment in $C_{i}$ connecting $v_{i}$ to $v_{i+1}\left(v_{1}\right.$ for $\left.i=4 n\right)$. Then the $c_{i}$ form a cycle $C$ without crossings (since any two non-adjacent segments of $C$ belong to disjoint convex sets). Now we can extend $\ell_{i}$ in $L_{i} \cup C_{2 i}$ so it connects to $v_{2 i-1}$ and in $L_{i} \cup C_{4 n-2 i+2}$ so it connects to $v_{4 n-2 i+1}$ without crossing $C$. Pick vertices $t_{i}^{1} \in S_{i}^{1} \cap S_{i}^{2}$ and $t_{i}^{2} \in S_{i}^{2} \cap S_{i}^{3}$. We can connect $t_{i}^{1}$ by a curve $s_{i}^{1}$ in $S_{i}^{1} \cup C_{2 i+1}$ to $v_{2 i}$ and $t_{i}^{2}$ by a curve $s_{i}^{3}$ in $S_{i}^{3} \cup C_{4 n-2 i+1}$ to $v_{4 n-2 i}$ without crossing any of the curves we have already constructed; finally, we can connect $t_{i}^{1}$ to $t_{i}^{2}$ within $S_{i}^{2}$ by a curve $s_{i}^{2}$ not crossing any other curve except, possibly, $\ell$. Now extend the curves we have constructed slightly, so that shared endpoints become crossing points. The resulting curves fulfill Lemma 1 with condition $\left(i^{\prime}\right)$ in place of $(i)$ : $\left(i^{\prime}\right)$ is true, since $L$ intersects $S_{i}^{2}, i \in[n-1]$, and none of these sets intersect $C$, so they must all lie on the same side of $C$. Now Lemma 1 allows us to conclude that $\ell$ is crossed by $\left(\ell_{i}\right)_{i \in[n]}$ in order $\ell_{1}, \ldots, \ell_{n}$ or the reverse of that order.

Proof (Theorem (3). It it easy to see that the problem lies in $\exists \mathbb{R}$. Suppose we are given a simple arrangement $\mathcal{A}$ of pseudolines. As earlier, we think of the arrangement as a set of simple, $x$-monotone curves.

Let $D$ be a disk which contains all the crossings of the pseudolines in its interior. Cut all the pseudolines at the boundary of $D$ and let their order of intersection with the boundary be $A_{1}, \ldots, A_{n}, A_{1}, \ldots, A_{n}$. Add sets $\left(B_{i}\right)_{i \in[2 n]}$, required to intersect cyclically: $B_{i}$ with $B_{i+1}$ and $B_{2 n}$ with $B_{1}$, with no other intersections, and sets $\left(H_{i}\right)_{i \in[2 n]}$, so that $H_{i}$ intersects $B_{i}$, and $A_{i}$ if $i \leq n$ and $A_{i-n}$ otherwise. Now for each of the $A$-, $B$ - and $H$-sets add the ordering gadget described in Lemma 2 Call the resulting intersection graph $G_{\mathcal{A}}$. (We will only make use of the ordering gadgets for $\left(A_{i}\right)_{i \in[n]}$, but we need to add them in such a way that they allow for the intersections with the other sets as well.) 
If $\mathcal{A}$ is stretchable, then the intersection graph we specified is realizable by convex sets (actually by line segments).

So suppose there is a drawing of convex sets realizing $G_{\mathcal{A}}$. Pick a vertex $u_{i} \in B_{i} \cap B_{i+1}$ for $i \in[2 n-1]$, and $u_{2 n} \in B_{2 n} \cap B_{1}$, and let $b_{i}$ be a straightline segment between $u_{i}$ and $u_{i+1}\left(u_{1}\right.$ for $\left.i=2 n\right)$. Then the $b_{i}$ form a cycle $B$ (without crossings). None of the $A_{i}$ intersect any of the $B_{j}$ so all $A_{i}$ must be on the same side of $B$. For each $A_{i}, i \in[n]$, pick a straight-line segment $\ell_{i}$ that starts in $A_{i} \cap H_{i}$ and ends in $A_{i} \cap H_{i+n}$. We claim that any two $\ell_{i}$ cross each other: each $\ell_{i}$ can be extended through $H_{i}$ and $H_{i+n}$ to connect to the cycle $B$. But then since two $\ell_{i}$ connect to alternating endpoints along $B$ and both curves are on the same side of the cycle, the curves must cross; since the $H_{i}$ do not intersect each other, that crossing must occur along the straight-line segments $\ell_{i}$.

Now Lemma 2 implies that the order of crossings along each $\ell_{i}$ is either the original order or the reversed order; however, since the order of intersection with $D$ is fixed by the cycle $B$, either all those orders are in the original order, or they are all reversed. But then, in either case, $\mathcal{A}$ is stretchable.

The claim about exponential precision again follows because the reduction we gave is geometric.

\section{Topological Inference}

Topological inference problems ask whether a specification of topological relationships can be realized by regions. The problems vary by what type of relationships (e.g. "contained in" and "disjoint with") and predicates (e.g. "connected", "convex") are available and what types of regions belong to the universe of discourse (2-dimensional, 3-dimensional, closed, regular, connected). For the current discussion we will restrict our universe to regular regions in the plane, not necessarily connected, where a region is regular if it is the closure of its interior.

There is a standard set of topological relationships, called RCC8, from the region connection calculus, that, in some sense, cover all possibilities of how two regions can be related to each other; the relations are, "disconnected" (DC), "externally connected" (EC), "equal" (EQ), "partially overlapping" (PO), "tangential proper part" (TPP), "tangential proper part inverse" (TPPi), "non-tangential proper part" (NTPP), and "non-tangential proper part inverse" (NTPPi), for details see 617. Other relations can be defined from the basic relations, for example "proper part" (PP) is the disjunction of TPP and NTPP.

In the language of RCC8 a relationship between two regions is a constraint and the conjunction of several constraints a constraint network (we do not need to allow negation, since the 8 relations are exhaustive (at least one of them has to hold). These are special cases of topological expressions, that is, Boolean formulas involving the 8 relations (typically excluding negation, since it is not necessary). We say a topological expression is realizable, if it is realized by regular regions in the plane. 
The problem of determining whether a constraint network (or a topological expression in general) is realizable lies in NP and is NP-complete for the full set of relations, though there are tractable fragments [17, Section 6]. If we restrict the universe to connected regions, the problem remains $\mathbf{N P}$-complete, as shown in 20,22 .

If we add the predicate "convex" to the signature of topological expressions, then the problem becomes $\exists \mathbb{R}$-complete.

Theorem 4 (Davis, Gotts, Cohn [5]). RCC8 with convexity is $\exists \mathbb{R}$-complete, this remains true even if the signature is restricted to $E C, P P$ and "convex" or $P O, D C$ and "convex". In the second case the result remains true if the constraint network contains a constraint for every pair of regions (the constraint network is fully specified).

Davis, Gotts, Cohn [5] only show the first part (EC, PP, and "convex"), we show the second part (restriction to PO, DC and "convex") here.

Proof. PO, DC and "convex" are enough to express that a graph $G$ is the intersection graph of convex regions in the plane (we require every region to be convex, so we are not bothered by the disconnected regions contained in the universe), which, together with Theorem 3 suffices to establish $\exists \mathbb{R}$-hardness. Note that we specify for every pair of regions whether they overlap (PO) or are disjoint (DC), so the resulting constraint network is fully specified. Davis, Gotts, Cohn [5] show that the problem lies in $\exists \mathbb{R}$.

Remark 4. If we restrict the universe of discourse to connected sets, then it is not immediately obvious that the realizability problem (with convexity) remains in $\exists \mathbb{R}$ : the issue at stake is that in this case the realizability problem without convexity is equivalent to the string graph problem, for which membership in NP is not trivial 22 20].

\section{References}

1. Bienstock, D.: Some provably hard crossing number problems. Discrete Comput. Geom. 6(5), 443-459 (1991)

2. Björner, A., Vergnas, M.L., Sturmfels, B., White, N., Ziegler, G.M.: Oriented matroids, 2nd edn. Encyclopedia of Mathematics and its Applications, vol. 46. Cambridge University Press, Cambridge (1999)

3. Bürgisser, P., Cucker, F.: Counting complexity classes for numeric computations. II. Algebraic and semialgebraic sets. J. Complexity 22(2), 147-191 (2006)

4. Canny, J.: Some algebraic and geometric computations in pspace. In: STOC 1988: Proceedings of the twentieth annual ACM symposium on Theory of computing, pp. 460-469. ACM, New York (1988)

5. Davis, E., Gotts, N.M., Cohn, A.G.: Constraint networks of topological relations and convexity. Constraints 4(3), 241-280 (1999)

6. Egenhofer, M.J.: Reasoning about binary topological relations. In: Günther, O., Schek, H.-J. (eds.) SSD 1991. LNCS, vol. 525, pp. 143-160. Springer, Heidelberg (1991) 
7. Garey, M.R., Johnson, D.S.: Crossing number is NP-complete. SIAM Journal on Algebraic and Discrete Methods 4(3), 312-316 (1983)

8. Goodman, J.E., Pollack, R., Sturmfels, B.: Coordinate representation of order types requires exponential storage. In: STOC 1989: Proceedings of the twenty-first annual ACM symposium on Theory of computing, Seattle, Washington, United States, pp. 405-410. ACM, New York (1989)

9. Hliněný, P.: Crossing number is hard for cubic graphs. J. Combin. Theory Ser. B 96(4), 455-471 (2006)

10. Kratochvíl, J.: Personal communication

11. Kratochvíl, J., Matoušek, J.: Intersection graphs of segments. J. Combin. Theory Ser. B 62(2), 289-315 (1994)

12. Kratochvíl, J., Pergel, M.: Geometric intersection graphs: do short cycles help (extended abstract). In: Lin, G. (ed.) COCOON 2007. LNCS, vol. 4598, pp. 118 128. Springer, Heidelberg (2007)

13. Kyncl, J.: The complexity of several realizability problems for abstract topological graphs (unpublished manuscript) (based on Graph Drawing 2007 paper)

14. Mnëv, N.E.: The universality theorems on the classification problem of configuration varieties and convex polytopes varieties. In: Topology and geometry-Rohlin Seminar. Lecture Notes in Math., vol. 1346, pp. 527-543. Springer, Berlin (1988)

15. Pach, J., Tóth, G.: Thirteen problems on crossing numbers. Geombinatorics 9(4), 194-207 (2000)

16. Pelsmajer, M.J., Schaefer, M., Štefankovič, D.: Crossing number of graphs with rotation systems. In: Hong, S.-H., Nishizeki, T., Quan, W. (eds.) GD 2007. LNCS, vol. 4875, pp. 3-12. Springer, Heidelberg (2008)

17. Renz, J.: Qualitative spatial reasoning with topological information. In: Renz, J. (ed.) Qualitative Spatial Reasoning with Topological Information. LNCS (LNAI), vol. 2293, p. 207. Springer, Heidelberg (2002)

18. Richter-Gebert, J.: Mnëv's universality theorem revisited. Sém. Lothar. Combin. 34 (1995)

19. Schaefer, M.: The real logic of drawing graphs (unpublished manuscript)

20. Schaefer, M., Sedgwick, E., Štefankovič, D.: Recognizing string graphs in NP. J. Comput. System Sci. 67(2), 365-380 (2003); Special issue on STOC 2002, Montreal, $\mathrm{QC}$

21. Schaefer, M., Štefankovič, D.: Fixed points, nash equilibria, and the existential theory of the reals (unpublished manuscript)

22. Schaefer, M., Štefankovič, D.: Decidability of string graphs. J. Comput. System Sci. 68(2), 319-334 (2004); Special issue on STOC 2001, Crete, Greece

23. Shor, P.W.: Stretchability of pseudolines is NP-hard. In: Applied geometry and discrete mathematics. DIMACS Ser. Discrete Math. Theoret. Comput. Sci., vol. 4, pp. 531-554. Amer. Math. Soc., Providence (1991) 